\title{
ANÁLISIS DE LOS DISEÑOS EN LOS HIPOGEOS DEL PARQUE ARQUEOLÓGICO DE TIERRADENTRO, CAUCA, COLOMBIA
}

\section{HYPOGEA DESIGN ANALYSIS OF THE TIERRADENTRO AROUEOLOGICAL PARK, CAUCA, COLOMBIA}

\author{
Armando Aroca Araújo ${ }^{1}$ \\ ${ }^{1}$ Licenciado en Matemáticas y Física. Magíster en Educación Matemática. Profesor de Tiempo Completo, Universidad del \\ Atlántico, Barranquila, Colombia, armandoaroca@mail.uniatlantico.edu.co
}

Rev. U.D.C.A Act. \& Div. Cient. 16(2): 525-534, Julio-Diciembre, 2013

\section{RESUMEN}

Se presenta una aproximación a la lógica de construcción que dio origen a los diseños de la pintura mural, al interior de los hipogeos, del Parque Arqueológico de Tierradentro, siendo tal vez uno de los vestigios geométricos más representativos del patrimonio matemático colombiano. En particular, se analizarán algunos diseños de hipogeos ubicados en las estaciones de Segovia, El Duende, Alto de San Andrés y El Aguacate, que fueron elaborados en las paredes, techos, pilastras, nichos y columnas. El enfoque de análisis de los diseños no responde a la imposición de la validación de este pensamiento por medio de la validación de teoremas o definiciones de la matemática académica.

Palabras clave: Geometría precolombina, pintura mural, osarios, lógica de construcción, etnomatemática, hipogeos de Tierradentro.

\section{SUMMARY}

An approach to the logic of construction that led to the mural design, inside the hypogea of the Tierradentro Archaeological Park, being these perhaps one of the most representative traces of the geometric mathematical Colombian heritage, is presented. In particular, some designs of the hypogea stations located at Segovia, El Duende, Alto de San Andrés and El Aguacate that were elaborated on the walls, ceilings, pillars, niches and columns, are analyzed. The focus of the analysis of these designs does not respond to the imposition of the validation of theorems or definitions of academic mathematics.

Key words: Pre-Colombian geometry, mural painting, ossuaries, construction logic, ethnomathematics, hypogea of Tierradentro.

\section{INTRODUCCIÓN}

Presentación y los propósitos del análisis: El Parque Arqueológico de Tierradentro está localizado en la Cordillera Central de Colombia, esencialmente, en las estribaciones de la población de San Andrés de Pisimbalá, del departamento del Cauca. Estos vestigios arqueológicos, considerados por antropólogos o arqueólogos como tumbas secundarias u osarios, fueron declarados, según Unesco (1995), como Patrimonio de la Humanidad y están conformados por cinco estaciones, donde cuatro aglutinan más de 70 hipogeos. Estos hipogeos, han sido analizados desde diferentes perspectivas, antropología, arqueología, arquitectura y artes, de cuyas investigaciones se podrían destacar Burg (1936), Pérez de Barradas (1937) y los diversos informes y dibujos linealizados por Hernández de Alba (1936a, 1936b, 1948), Celis (1943), Nachtigall (1956), Ayala (1983; 1986), Gamboa (1983; 1985), Chávez \& Puerta (1986). Se destaca, además, lo indicado por la Unesco (1995) y Sevilla (2008, 2009), donde este último presenta la sistematización organizada de los hipogeos. En estas investigaciones o análisis se presentan expresiones, como "decoración geométrica", "motivos geométricos", "pintura mural”, "geometría”, "geometría lineal", "figuras geométricas", "simetría". ¿Pero a qué se hace referencia concretamente cuando se emplean estas expresiones? Hasta el momento no se ha hecho un estudio sistemático que tome como objeto de estudio la configuración geométrica que aún se nota en los hipogeos, excepto descriptivo.

\section{MATERIALES Y MÉTODOS}

El tipo de investigación, se enmarca dentro de la arqueología cognitiva, descrita en Rivera (2005), pues lo que se pretendió fue decodificar la lógica del diseño plasmado en la pintura mural de los hipogeos. Esto conllevó a establecer regularida- 
des o patrones específicos, que permitieron comprender un poco, para este caso, lo que nuestra lógica entienda por pensamientos geométrico y métrico, que empleó dicha cultura. Sumado a esto, el análisis documental de las investigaciones descritas en la introducción, la observación misma al interior de los hipogeos, el análisis de imágenes fotográficas y la elaboración de fichas de registros que se emplearon en cada estación del parque, conllevaron a establecer los resultados que se describen a continuación.

\section{RESULTADOS Y DISCUSIÓN}

Nachtigall (1956) plantea que la diferencia que existe entre las tumbas, en cuanto a su decoración, se debe a diferencias sociales en clases de jefes; sin embargo, el empleo de nociones o de concepciones geométricas en dichas decoraciones era indistinto a estas diferencias sociales, pues lo que se incrementaba, debido a la superficie a cubrir del hipogeo, era la reproductibilidad en el diseño, es decir, era más profusa la configuración. ¿̇Acaso aquellos pintores que decoraban la tumba de un gran jefe no harían lo mismo con los hipogeos de sus familiares, suponiendo que estos artesanos no estaban en el rango de servidores sino del pueblo, como lo plantea el mismo Gamboa (1983), que les daría así el derecho a hacer entierros secundarios? Otra pregunta, entonces sería necesaria, ċera esta práctica de apropiación colectiva o solamente era de un grupo de personas que lo hacían? Si es un sí, entonces, las nociones geométricas eran las mismas y no dependían de la estratificación social ni tampoco de la profundidad y el tamaño del hipogeo, pues las nociones, que dependían del tiempo evolutivo de su arquitectura, cuya transformación presentó Ayala (1986), serían aplicadas, sea en pequeñas superficies o grandes. A mayor profundidad y tamaño, más columnas y pilastras, podría tener el hipogeo y, como consecuencia, más superficie habría por cubrir con blanco y, luego, decorar con negro, rojo o amarillo ocre.

Las nociones geométricas en la configuración de los diseños de los hipogeos: En la actualidad, se puede percibir que todos los hipogeos presentan deterioro en su pintura mural, en porcentajes que oscilan entre el $20 \% \mathrm{y}$, posiblemente, el $100 \%$. En cada estación, se pueden encontrar nociones geométricas que la caracteriza, en cuanto a formas, individualización de lo representado y combinación de colores; pero en la mayoría de estos diseños, en particular en aquellos donde el romboide tiene gran reproductibilidad, se puede notar el desarrollo de cinco nociones que dan origen al diseño en general. Lo primero que tal vez dibujaba el artesano o dibujante eran las franjas de separación, que en algunos hipogeos eran reemplazados por los relieves de división de la misma arquitectura, las cuales, delimitan la zona de configuración mínima, y si esta lo desarrolla, se establece un patrón figural, que es tomado para la reproductibilidad que genera el diseño general. Como se puede ver en la figura
1, dicho patrón figural podría verse también como la mitad del que se indica como un "triángulo expandido" y no el rombo o romboide, pero se ha tomado éste último por el simbolismo que tiene dicha figura en los diseños que recubren el interior de la mayoría de los hipogeos de Tierradentro. La configuración mínima, incluye entonces, tanto al patrón figural como la direccionalidad del diseño, en otras palabras, si el inicio de éstos fue de abajo hacia arriba o viceversa, o del centro hacia arriba o hacia abajo, o de manera horizontal ascendente o descendente, o de izquierda a derecha o viceversa. Estas ideas, incluso, podrían estar sujetas a si participó una o más personas en el diseño, pues no era una actividad de fuerza, como la excavación del hipogeo, era una actividad de pocas personas que desarrollaban el diseño geométrico o métrico; un ejemplo es la figura 5; más la obtención de pinturas, el manejo del "pincel" o "brochas", desarrollo de diseños mentales o modelamiento, que en algunos momentos tuvieron que tener en cuenta para llegar a las partes más incomodas de dibujar y pintar como el techo o la cornisa.

La direccionalidad, definida por las franjas de separación, puede ser horizontal, oblicua o vertical. Estas franjas de configuración pueden tener o no en su interior otra configuración, como las que se aprecian en la tumba NA11, donde se incluyen romboides, círculos negros o círculos rojos; en cambio, en las estaciones bajas, esta ocupación de la superficie no se encuentra. La figura 1, muestra la deconstrucción de las nociones.

El papel del color en la configuración: Según Sevilla (2009), fueron cuatro los colores empleados en los diseños: el negro (de mayor predominación visual), el rojo, el amarillo ocre (el de menor uso) y el blanco (que servía de base, pero implicaba resaltar la forma). Chávez \& Puerta (1986) indican que la pintura blanca muestra todos los caracteres de una arcilla blanca (que pudo ser aplicada con la mano o un artefacto plano). Se trata de un material muy plástico. La roja, es simplemente un ocre rojo, fácilmente disgregable al ser tratado por el agua. La pintura negra es un material insoluble al agua; mediante un simple lavado con agua, se separa en forma de escamas negras. Funde por debajo de seiscientos grados centígrados y durante la fusión, su color varía hacia el blanco. Se trata de un material compuesto de pizarra negra arcillosa.

Para este caso, las nociones geométricas se vehiculizaron, entonces, por medio de la pintura y, en particular, por una predominación de trazos lineales, hechos en superficies planas o cóncavas, que les confería una especie de propiedad de curva en la superficie. El color, interpretando a Velandia (1994), asumió no solamente un papel ornamental sino que dio forma y, por ello, se puede observar que existe un orden, que se podría decodificar para dar una aproximación a la realización de los diseños. 


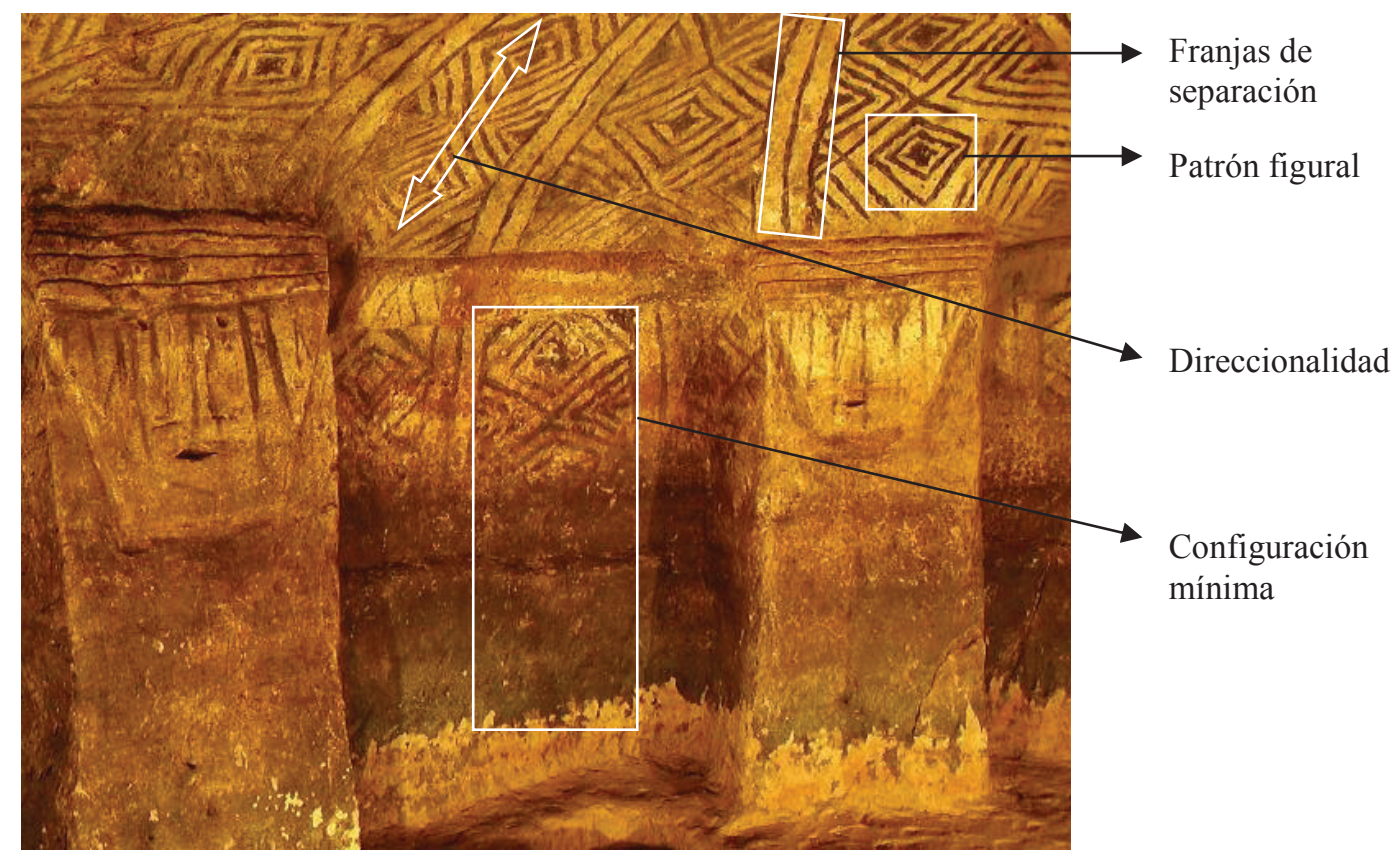

Figura 1. Algunas nociones geométricas en la configuración de los diseños.

Tomando como referencia el modelo propuesto por Velandia (1994), para la combinación del color en la estatuaria de San Agustín, se podría plantear las fórmulas de combinación de colores que se encuentran en los hipogeos de Tierradentro. Esto lo muestra la figura 2.
Velandia (1994) determinó que la cultura de San Agustín empleó cuatro colores: los tres que emplearon en Tierradentro más el amarillo ocre y pudo establecer nueve fórmulas de combinaciones. En Tierradentro, solamente tomando los romboides concéntricos al interior de los hipogeos, que em-

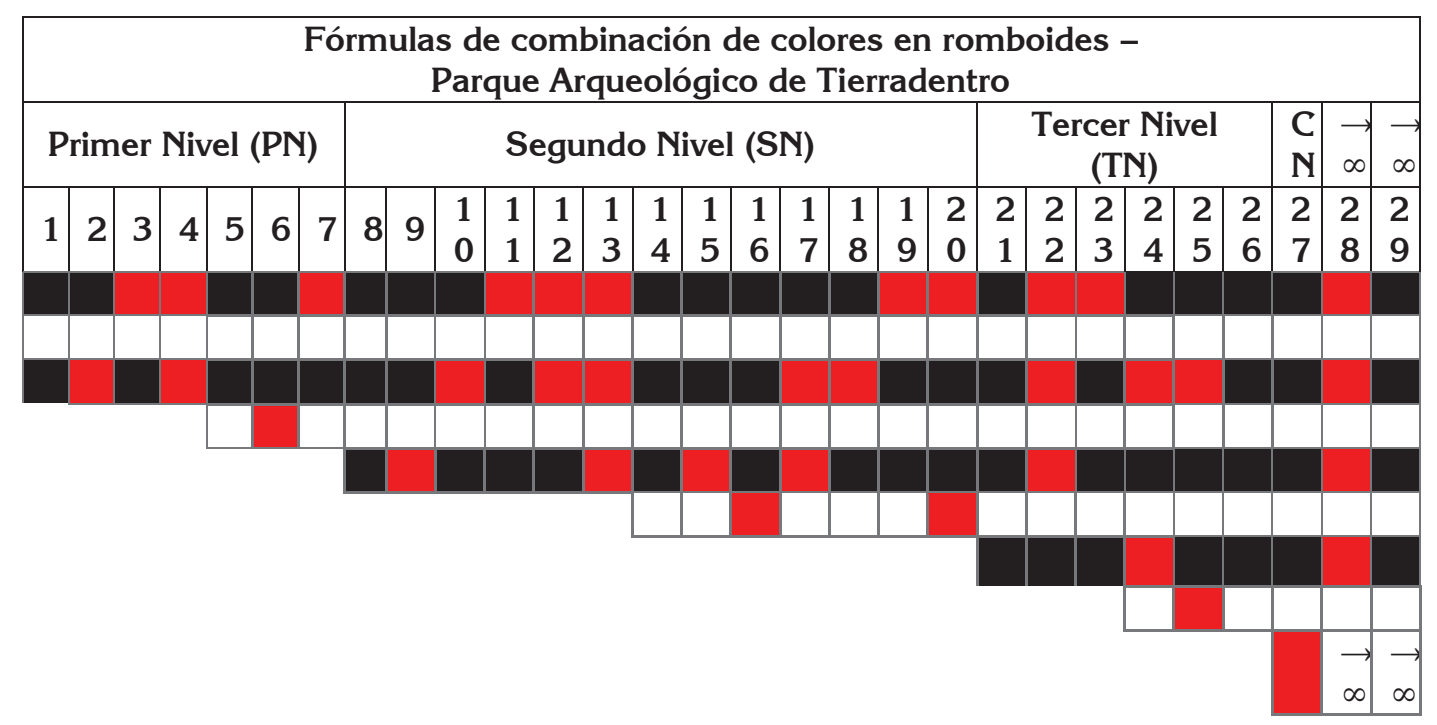

Figura 2. Las fórmulas de combinación de colores en los patrones figurales de los hipogeos, del Parque Arqueológico de Tierradentro. 
plearon tres colores, se pudo determinar que por ahora son 29 fórmulas, como lo muestra la figura anterior.

Después de determinar las fórmulas que se encuentran en la actualidad en cada estación, dadas por la figura anterior, se pudo concluir que la única fórmula que se puede encontrar en cada una de ellas es la F8, pero que no hay ninguna que sea transversal a cada uno de los hipogeos.

Las franjas de separación, al parecer el inicio del diseño: $\mathrm{Al}$ parecer lo primero que dibujaba el artesano en las paredes, en muy pocas columnas y pilastras y en la parte inicial del techo hasta el remate con la cornisa, eran las franjas de separación. Inicialmente, se deben hacer las franjas de separación en su forma básica, tal vez solamente dos franjas negras o una franja roja, pues estas delimitan para dar inicio a la configuración mínima, es decir, el papel inicial de estas franjas es de delimitación de la superficie en sectores de desarrollo creativo y, en su mayoría, con el empleo de romboides concéntricos.

Da la impresión que, para ubicar algunas franjas de separación, los dibujantes empleaban algún instrumento calibrado, cuya longitud era tomada para empezar las líneas de las franjas de separación. Se pudo constatar, a partir de algunas fotos tomadas frontalmente, que esta separación es muy precisa, bastaba con colocar la imagen como plantilla en un programa de análisis de imágenes para verificar que muchas medidas son coincidentes. Entonces, el empleo de algo, como patrón de medida, por ejemplo, una vara o un tronco delgado, consistente y de madera, es una posibilidad real. El empleo únicamente de dedos era dispendioso y por medio de la estimación ad óculos, tal vez les generaría problemas de semejanza o congruencia, que no se ven al inicio de algunas configuraciones mínimas. En comparación, sabiendo que desarrollaron un alto grado de conocimientos en orfebrería y en alfarería, más en ingeniería y en arquitectura, se valida más aún esta posibilidad.

Se puede concluir que existen dos tipos de franjas de separación: aquellas que solamente incluyen líneas y cuyas fórmulas de combinación de colores son Blanco (B), Negro (N), Rojo (R) y Amarillo ocre (A), impares de 1 a 5, a saber: R, $\mathrm{N}, \mathrm{N}-\mathrm{B}-\mathrm{N}, \mathrm{N}-\mathrm{R}-\mathrm{N}, \mathrm{R}-\mathrm{A}-\mathrm{N}, \mathrm{N}-\mathrm{B}-\mathrm{N}-\mathrm{B}-\mathrm{N}, \mathrm{A}-\mathrm{B}-\mathrm{R}-\mathrm{B}-\mathrm{A}$ y N-B-R-BN. De estas fórmulas de combinación de colores, se precisa que tanto el amarillo ocre como el rojo no fueron empleados de manera popular como líneas externas en la franja de separación, fue de uso exclusivo, tal como se evidencia en el hipogeo NA36 y, en particular, en la estación El Aguacate. Un segundo grupo es aquel que al interior del par o terna de líneas incluye otros tipos de figuras planas, como círculos, circunferencias o cuadrados, muy visibles, de manera fragmentada, también en la estación El Aguacate, lo que les confiere una inversión significativa de pensamiento geomé- trico y tiempo en sus diseños, siendo en el hipogeo NA20, donde a las franjas de separación se les da un grado más de complejidad, pues asumen tanto el papel de éstas como de configuración mínima en la parte baja de la pared, al tener en su interior una sucesión vertical de rectángulos. Este es el único hipogeo donde se encuentran cuadrados al interior de ellas. El hipogeo NA29, en sus paredes, no tiene franjas de separación, pero no así en sus pilastras y estos diseños que se encuentran en dicha pared, no se repiten en las demás estaciones, que son una especie de sucesión horizontal de pequeñas líneas oblicuas, que se reflejan verticalmente. La figura 3, muestra algunas franjas de separación en dos estaciones.

El empleo del rojo y el negro en la estación de San Andrés es más notorio, no aparece el amarillo ocre, que es exclusivo de El Aguacate, pero aquí en la estación, el rojo se ve más que en otras estaciones, sin plantear que sea el dominante, color que era empleado para "embalsamar" a los muertos después de ser extraídos de la tumba primaria, para luego ser colocados en el osario o hipogeo. Da la impresión por el tipo y el ancho de los trazos, que tanto en esta estación como en la estación de Segovia, en particular en los hipogeos NSA1 y en el enigmático NS2, se haya empleado una especie de brocha, que pudo tener la misma forma del pincel, pero de mango más grueso y, por ende, cabeza, porque hacer algunas de estas franjas no responden al mismo trazo de los romboides y en sus bordes aún se aprecia linealidad. Solamente cuatro tipos de franjas de separación horizontal están en el hipogeo NS2; los demás, presentan únicamente dos tipos de franjas de separación verticales.

¿Si los restos de los cuerpos de los hombres y de las mujeres eran colocados de manera separada, los diseños geométricos se hacían por igual en los nichos o cerca de los depósitos donde residía la osamenta? Visto solamente de esta manera, no es fácil aceptar la tesis de Ayala (1986), que la decoración tiene como objeto ayudar a representar los elementos de la estructura del hipogeo. Si se tratara únicamente de resaltar, otras técnicas tal vez serían empleadas, pero el tiempo asociado a la inversión de pensamiento espacial, geométrico y métrico no hubiese sido tanto. Es claro, entonces, que existió simbolismo más que un mero papel de hacer contraste a partes de la estructura interna del hipogeo. Existen evidencias en todas las estaciones que los pintores centraron más su atención en lo que dibujaban que en el elemento mismo de la estructura del hipogeo. Como se puede evidenciar en las técnicas: fórmulas de combinación, linealidad, direccionalidad, medición, semejanzas y formas que, a continuación, se seguirán describiendo.

Patrones figurales: La serpiente, que es el motivo del que más tratan en su alfarería los pobladores que construyeron los hipogeos, según Gamboa (1985), se reduce a formas, 


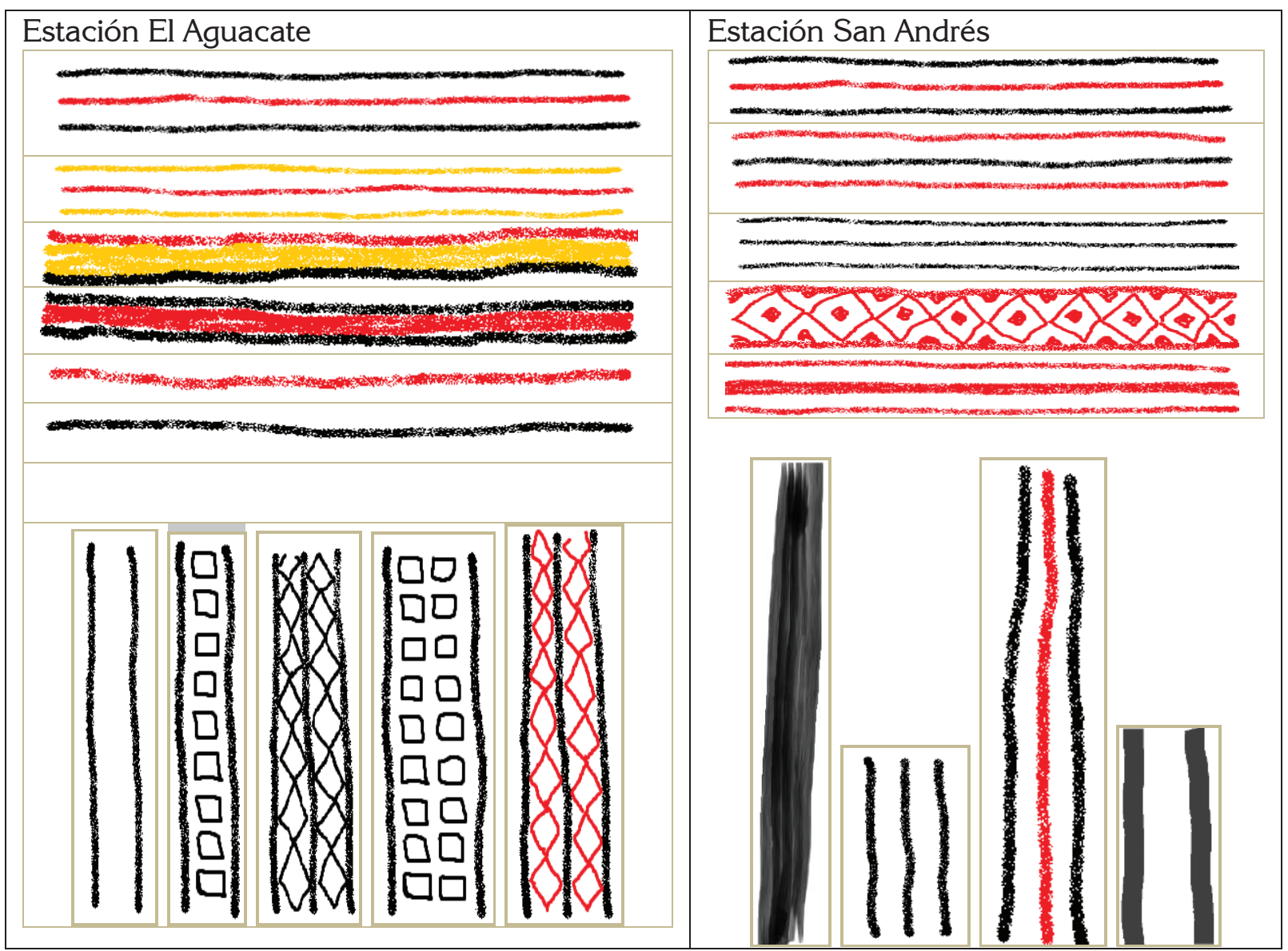

Figura 3. Algunas franjas de separación en las estaciones El Aguacate y San Andrés.

como el romboide, el rectángulo y el cuadrado. Siendo el romboide, como elemento representativo de la serpiente de cascabel, para esta cultura fue la coral, el simbolismo del espacio - tiempo, pero según Chávez (1981), en culturas actuales, hay coincidencia en la significación de la serpiente como fertilidad y destrucción, como principio dual de vida y muerte, como elemento de unión entre diversos mundos. Teniendo el romboide esta connotación simbólica, a las franjas de separación las suceden en el diseño dichas figuras, las cuales, se denominarán patrones figurales, como se definió en Aroca (2009): es la forma mínima para la reproductibilidad. Lo primero es preguntarse si los rombos son concéntricos como muchos antropólogos o arqueólogos lo han manifestado, ¿̇son concéntricos o tienen interdependencia o se amplifican o contraen en torno al centro definido a veces por una estrellita? Analicemos la figura 4, que muestra ciertos detalles en la configuración de los romboides.
Se puede notar que los trazos rojos que están en el centro de la imagen no se dibujaron de manera continua, como sucede en algunas pilastras o columnas, aunque esta técnica no es general en todo el Parque, sino que el dibujante primero hizo el romboide completo, trazó su poligonal, es decir, el trazo 1 no es continuo con el trazo 4. Terminado el trazo 1 , en el vértice, se sigue con el trazo 2 o viceversa, si esto es así, como aparentemente lo es, tiene unas implicaciones en la forma de hacer los diseños del Parque, en particular, en lo que se ha denominado romboides concéntricos. Si los romboides aparecen en pilastras o en columnas, responden a una técnica diferente, que podríamos determinar por las intercepciones de dos grupos de familias de paralelas oblicuas inversas entre sí. Si los romboides aparecen en las paredes o en las cornisas, al parecer se hacen amplificando el romboide central (que es de una clase distinta a las poligonales, pues al parecer representa una estrella, como lo plantea 


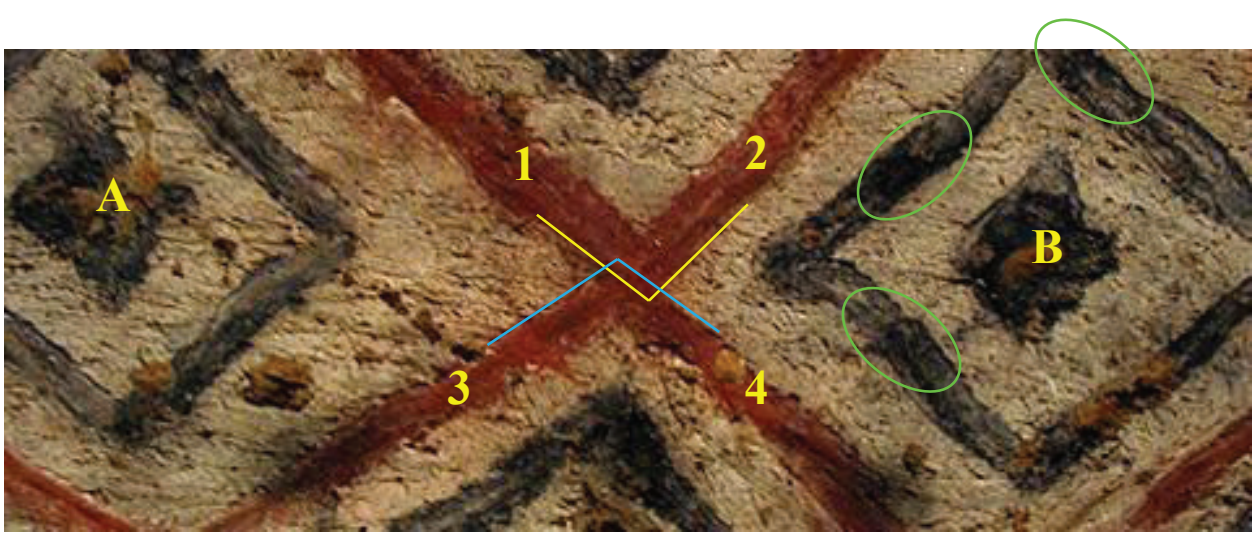

Figura 4. Análisis de concentricidad o amplificación de romboides. Tomada de http://www.icanh.gov.co/index.php?idcategoria= 2560.

Pérez de Barradas (1937), el más próximo al centro común que, por lo general, define una superficie, figuras A y B y no una poligonal, como lo hacen los exteriores; lo anterior se puede validar viendo que estos romboides centrales tienen una secuencia, están sobre una misma línea, mientras que los exteriores, por ejemplo, si se va dibujando hacia arriba, se van contrayendo o deformando, pero el central se mantiene en la línea de la dirección de la reproductibilidad, es entonces ese romboide central el que define, en muchos hipogeos, la configuración mínima. El hecho de que se amplifiquen, en este caso, conlleva a ser concéntricos, pero la visión arqueológica y antropológica da la impresión que los haya concebido siempre de afuera hacia adentro, cuestión que sería muy complicado para el diseño, sino se define dicho centro, en algunos casos.

Los romboides centrales, que contienen dicho centro, fueron hechos al parecer en cinco trazos: cuatro curvos hacia dentro y un pincelazo o con el dedo que rellenaría su región central, lo que en definitiva les da una apreciación de clase distintas; de hecho, en muchos casos, el pintor plasmó la intención de no dejar lineal sino convexa los contornos de esta figura. Esto se puede pensar por la curva que tienen sus lados y porque el color de estos es muy homogéneo, más tinta en trazos muy cortos, a diferencia de las poligonales, que aún hoy, más de 1.400 años, se les nota la superposición del trazo, cuando el dibujante volvía con su pincel o algo untado, como se ve alrededor del romboide B. Estos trazos, entonces, eran muy pequeños, lo que se puede sustentar en que empleaban ollas pequeñas de barro, a manera de cuerno, para depositar las pinturas, como se describe en Ayala (1986); incluso, desde la misma capacidad del recipiente, se podría pensar que había más atención a los trazos. En cuanto a transformaciones de posición, cuando se emplean los romboides, no se evidencian giros, reflexiones, ni deslizamientos, solamente traslaciones y se debe a la individualiza- ción de la configuración mínima, pero las transformaciones de más uso, cuando se emplea tanto el romboide, el triángulo y el cuadrado en los hipogeos, son las transformaciones de forma, tanto semejanzas como amplificaciones.

Se precisa, ante la linealidad alcanzada por los contornos, excepto por la hipotenusa de los triángulos rectángulos que custodian un rostro en el hipogeo NS2, que estos diseños fueron hechos a mano alzada, pero que hay ciertos indicios donde, al parecer, se empleó algún instrumento que sirvió como patrón de medida para delimitar las distancias entre franjas de separación, es decir, para delimitar puntos exactos de inicio de las líneas que conforman las franjas de separación y las transversales que generan los romboides o para hacer otro tipo de diseño, como el que se evidencia en el hipogeo NS2, donde hay siete distancias que, posiblemente, fueron medidas para hacer el dibujo (Figura 5).

Se puede suponer que las medidas a se marcaron tomando como referencia alguna parte del cuerpo o empleo de una vara; luego, se marcaba la pared, por ejemplo, con el otro extremo del pincel. La explicación del por qué a es mayor que c, al parecer responde a efectos de dibujar con comodidad al estar de pie, no hubiese sido lo mismo si $\mathbf{c}>$ a. Al tener como referencia la franja de separación superior y que al parecer la inferior fue hecha después por su falta de horizontalidad y de vínculo simétrico con el resto de la distribución, se procedió a establecer el tamaño de los triángulos, donde presuntamente las ternas fueron pintadas de afuera hacia dentro. Al meterse éstas dentro de una elipse, se nota que la terna de triángulos del lado izquierdo ocupa más superficie que la terna de la derecha, lo que implica que no se tuvo en cuenta los tamaños entre sí, excepto, por el empleo de la vista; sin embargo, se presenta una sorprendente coincidencia entre las distancias de los vértices inferiores de cada triángulo con el que le sigue hacia dentro, representadas por 


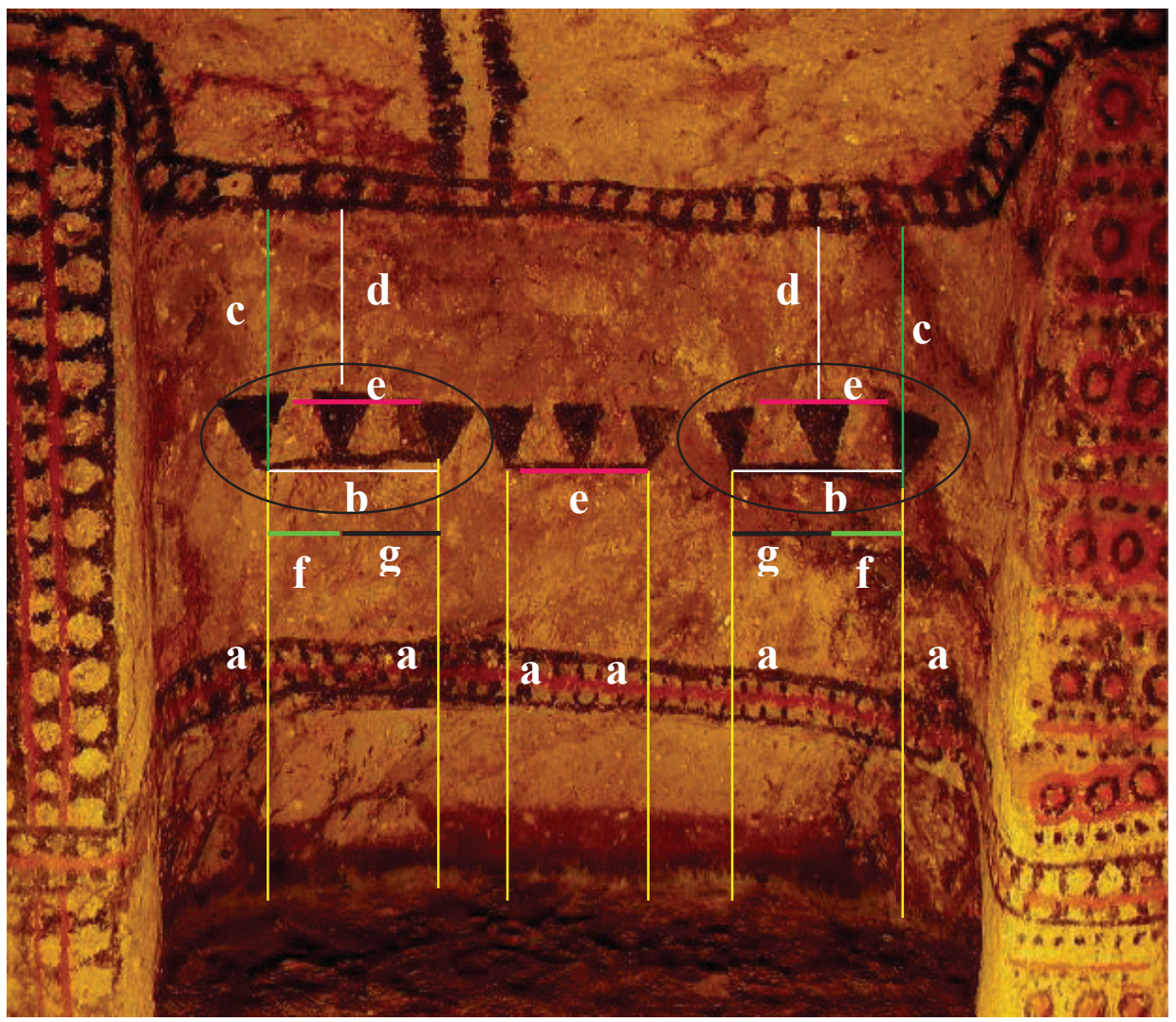

Figura 5. Algunas medidas casi congruentes encontradas en el hipogeo NS2.

las medidas $\mathbf{f}$ y g. Posteriormente, se tendrían en cuenta las medidas $\mathbf{d}$ y $\mathbf{e}$.

En el caso del romboide es pertinente precisar algunos aspectos. En ciertos casos no siempre fueron creados como unidad sino que aparecieron como consecuencia de alguna construcción geométrica, es decir, aparecían por la interceptación de la familia de líneas paralelas oblicuas hacia la izquierda, al interceptarse con la familia de líneas paralelas oblicuas hacia la derecha, como ya se había manifestado. En algunos casos y, en especial sobre pilastras, los puntos de inicio de estas familias, están separados a distancias prácticamente congruentes, lo que supone el empleo de un patrón de medida. Estos romboides manejan distintos grados de nivel de amplificación y de combinación, tanto cromática como de poligonales y superficies, como se puede ver en la figura 6, donde en cada celda se presentan, a manera de ejemplo, el código de un hipogeo, que contiene la combinación y el nivel de amplificación.

Las cornisas y los techos de los hipogeos y pedazos de la superficie que aún han soportado el embate del tiempo y descuido administrativo, debelan otros niveles de amplifica- ción de los romboides. Da entonces la impresión que existe un nivel infinito, tanto para el color negro como para el color rojo, sin combinarse entre sí y esto se encuentra en el techo, al interior de la cornisa, del hipogeo NS23. Da la impresión que esta fue la idea del dibujante, creando la sensación, mirando hacia arriba, que se amplifican ad infinitum, como se puede apreciar en Gamboa (1985).

¿Por qué es el romboide la figura plana de mayor empleo en las configuraciones geométricas de los hipogeos? Solamente, a manera de hipótesis, da la impresión que parte de la respuesta se encuentra en un hipogeo que, justamente, no tiene romboides en su configuración, el NS2. En una de las dos pilastras de este hipogeo, que es el único que tiene trazos circulares, con reproductibilidad y con direccionalidad y que además tiene una combinación única de colores, que no se encuentra en ninguno de los patrones figurales, rojonegro-rojo; tiene elementos de configuración geométrica, que pueden generar hipótesis desde lo simbólico, pues el número cuatro tiene representación muy explícita. En ambas pilastras, el cuatro se repite, tanto vertical como horizontal, desde la entrada; la pilastra de la izquierda tiene división vertical por medio de tres líneas rojas que, a su vez, subdivide la 


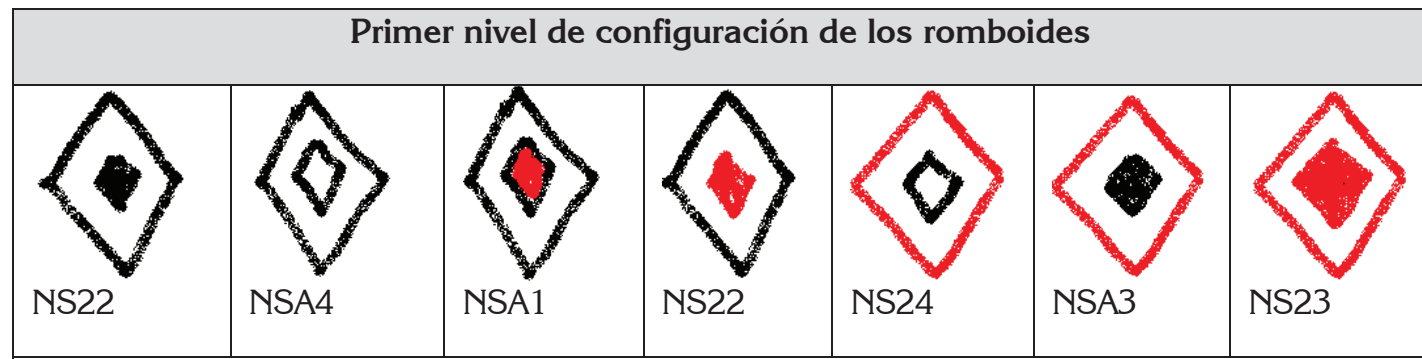

Hay un tipo de configuración descrita por Pérez de Barradas (1937), que no encontré, una aproximación de su descripción sería la siguiente:
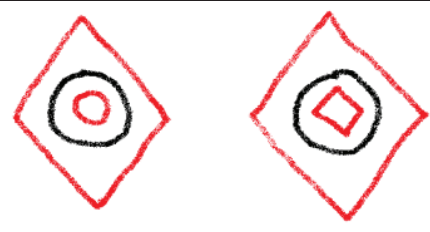

En algunas paredes o techos, se pudieron encontrar otras doce formas de combinación, que se podrían denominar de un segundo nivel, pues se incluía un nuevo romboide.

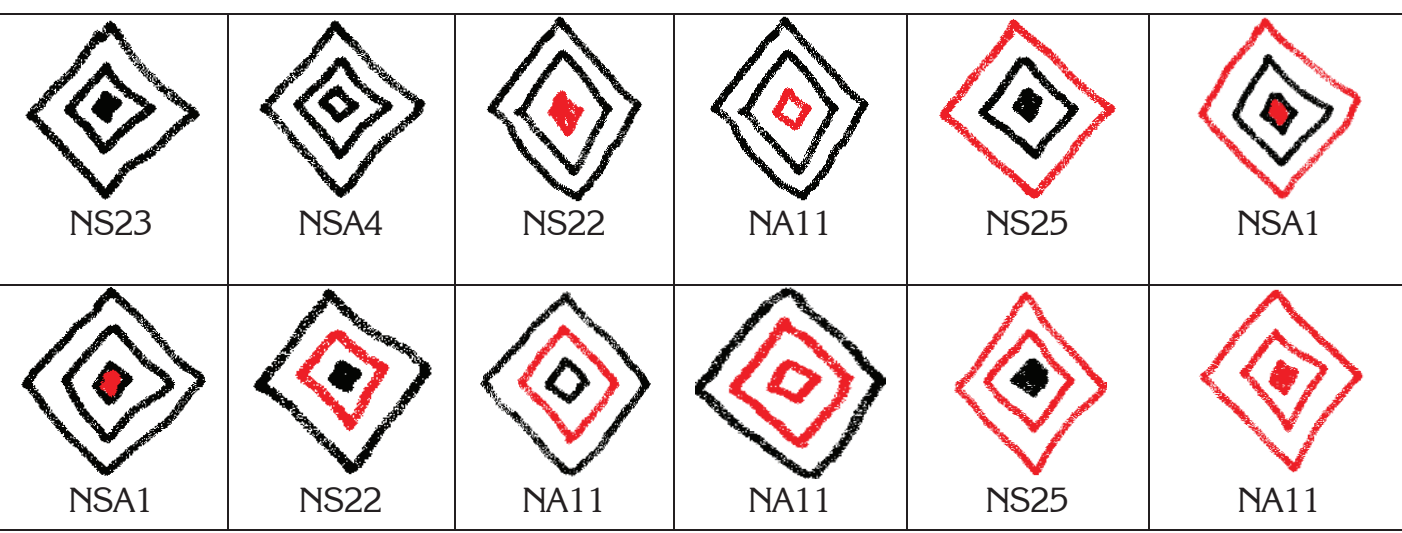

Un tercer nivel de configuración, se evidenciaron cinco combinaciones. Este tipo de combinación solamente se halló en paredes.

En el hipogeo NA39, los romboides están separados, no hacen parte de un mosaico, no
realzan ningún elemento de la estructura es el único hipogeo donde, al parecer, se
presenta esto. Cuarto nivel de amplificación.

Figura 6. Los patrones figurales en diversas configuraciones mínimas de los hipogeos. 
parte frontal en cuatro sub columnas de alta configuración; la pilastra de la derecha, también tiene claramente definido la representación del cuatro por medio de ocho grupos de cuatro círculos diseñados horizontalmente. También el techo del hipogeo está dividido en cuatro partes, por una franja de separación de trazos más gruesos de lo comúnmente empleado en otras estaciones. Pareciera que hubo la intención de representar el cuatro, pues cuando se entra a este hipogeo, se ve frontalmente, en las dos únicas pilastras y en el techo. Es probable que en él se encuentre representado el simbolismo del cuatro que, en la gran mayoría de los demás hipogeos, se asumió por la figura, que hoy llamamos romboide. También llama la atención que varias escaleras helicoidales en su remate tengan cuatro escalones lineales, como por ejemplo, los hipogeos NS21, NS9 y otros.

La configuración mínima: Definido por el artesano el patrón o los patrones figurales a emplear, pues la fórmula que empleaba en una configuración mínima no necesariamente sería la misma en la contigua, procedía entonces, después de establecidas las franjas de separación a pintar pequeños mosaicos, o formas que no necesariamente ocupaban toda la superficie, como lagartijas, lunas, romboides separados, entre otras. También se pudo observar que los romboides, que delimitaban con la configuración mínima, empezaban de manera similar en un extremo, pero en el opuesto no. Existen elementos para pensar que varios diseños se iniciaron de arriba hacia abajo, pues, en algunos casos, el vértice superior de un conjunto de cuatro romboides, coincidían plenamente en la parte superior, mientras en la parte de abajo nada coincidía, o sea, en este caso, el pintor no se iba fijando de una configuración mínima para hacer la otra contigua.

Definida entonces las configuraciones mínimas inferiores, se procedía a dibujar las configuraciones mínimas superiores, que se hacían de abajo hacia en el centro del techo, debido al mismo criterio ya expuesto y por los remates abruptos que se notan en algunos techos, esto porque las franjas de separación vertical tendían a cerrarse hacia dicho punto central del techo. En varios casos, el remate hacia al techo tiene mayores imprecisiones que el resto de las configuraciones mínimas, pues la altura tuvo incidencia y, en particular, la posición del cuerpo para pintar. Al no llegar directamente hasta la superficie superior del hipogeo tuvieron que montarse en algo, lo que implicaría tener que dibujar con los ojos mirando hacia arriba y tener el tronco en posición vertical, esto es una tarea demasiado extenuante para el cuello.

Reproductibilidad y direccionalidad: En la direccionalidad algunos romboides amplificados se hacían sea hacia la derecha o hacia la izquierda y otros seguían una dirección hacia arriba o hacia abajo; otros, de manera oblicua, hacia el centro del techo, que depende de la configuración mínima. La dirección del diseño en la configuración mínima se puede apreciar gracias al romboide central; por ejemplo, en el hipogeo ND1, hay una configuración mínima, donde los romboides tienen direccionalidad oblicua, los romboides centrales rojos. En una de las pilastras del hipogeo NSA4, se puede notar una intención de construir las líneas, de manera paralela, donde uno de los puntos para empezar sería el vértice superior izquierdo de la parte frontal; en consecuencia, el dibujante empezó a hacer los trazos de arriba hacia a abajo de manera oblicua hacia la izquierda. Por último, se dibujaba el romboide central, el que se asemeja a una estrella.

Para finalizar, el culto a la muerte permitió que esta cultura evolucionara en su pensamiento geométrico y métrico en cuanto al desarrollo de la pintura mural y fue la configuración de las superficies al servicio de la idolatración lo que les permitió, en este espacio de trabajo, sujeto a sus técnicas y herramientas propias al oficio, crear o dinamizar nociones que, con el paso del tiempo, irían evolucionando y que en el presente se constituyen un orgullo cultural para la Nación.

Conflicto de intereses: El manuscrito fue preparado y revisado por el autor, quien declara que no existe ningún conflicto de intereses, que ponga en riesgo la validez de los resultados presentados.

\section{BIBLIOGRAFÍA}

1. AROCA, A. 2009. Geometría en las mochilas arhuacas. Por una enseñanza de la geometría desde una perspectiva cultural. Ed. Universidad del Valle. (Santiago de Cali). 234p.

2. BURG, J. 1936. [Correspondencia recibida y enviada por Jorge Burg] [manuscrito]. Consulta de Sala. Biblioteca Luis Ángel Arango. 6p.

3. AYALA, L. 1986. Las tumbas pintadas de Tierradentro. En: Barney, E. (Org.) Historia del Arte Colombiano. Vol.I. Bogotá: Salvat Editores. p.188-212.

4. AYALA, L. 1983. Tierradentro, la Serranía de los Muertos. En: Barney, E. (Org.). Historia del Arte Colombiano. Vol.2. Salvat Editores. Bogotá. p.149-188.

5. CELIS, E. 1943. La arqueología de Tierradentro. Rev. Inst. Etnol. Nal. 1:117-130.

6. CHÁVEZ, A. 1981. Los animales mágicos en las urnas de Tierradentro. Museo de Artes y tradiciones populares. Bogotá. 109p.

7. CHÁVEZ, A.; PUERTA, M. 1986. Monumentos arqueológicos de Tierradentro. Talleres gráficos Banco Popular. Bogotá. 204p. 
8. GAMBOA, P. 1983. Tierradentro. Los constructores de hipogeos. Disponible desde Internet en: http://www. gamboahinestrosa.info/pdf/3\%20tierradentro.\%20 constructores\%20de\%20hipogeos.pdf (con acceso 20/03/2013).

9. GAMBOA, P. 1985. El arte de tierradentro. Disponible desde internet en: http:/gamboahinestrosa.info/ pdf/4.\%20el\%20arte\%20de\%20tierradentro.pdf (con acceso 20/03/2013).

10. HERNÁNDEZ DE ALGA, G. 1936a. San Andrés y Tierradentro [Manuscritos]: [Cuaderno de notas]. Cuaderno de notas. Consulta de sala. Biblioteca Luis Ángel Arango. 38p.

11. HERNÁNDEZ DE ALGA, G. 1936b. Notas arqueológicas. Exploración de una antigua sepultura en Tierra Adentro. [Manuscritos]. Archivo Gregorio Hernández de Alba. Estudios etnológicos. Consulta de sala. Biblioteca Luis Ángel Arango. 2p.

12. HERNÁNDEZ DE ALGA, G. 1948. Esquema arqueológico de Tierra Adentro [Manuscritos]. Consulta de sala. Biblioteca Luis Ángel Arango. 4p.

13. NACHTIGALL, G. 1956. Tierradentro. Studia. 1(10):21-55.

14. ORGANIZACIÓN DE LAS NACIONES UNIDAS PARA LA EDUCACIÓN, LA CIENCIA Y LA CULTURA -
UNESCO-. 1995 Disponible desde internet en: http:// portal.unesco.org/es/ev.php-url_id $=45692 \&$ url do $=$ do_topic\&url_section $=201 . h$ tml (con acceso 15/12/12).

15. PÉREZ DE BARRADAS, J. 1937. Arqueología y antropología precolombinas de Tierra Dentro. Imprenta Nacional. Bogotá. 100p.

16. RIVERA, Á. 2005. Arqueología cognitiva: Origen del simbolismo humano. Arco Libros. Madrid. 96p.

17. SEVILLA, E. 2008. Tierradentro: Les arts premiers y la jigra de la vida. Cali: Facultad de Artes Integradas, Universidad del Valle. Disponible desde internet en: www.tierradentro.info (con acceso el 14/02/13).

18. SEVILLA, E. 2009. Informe final del Proyecto Tierradentro, No. 398. Bogotá: Banco de la República, Fundación de Investigaciones Arqueológicas Nacionales, FIAN. Disponible desde internet en: www.tierradentro.info (con acceso 15/12/12)

19. VELANDIA, C. 1994. San Agustín. Arte, estructura y arqueología. Ed. Presencia. (Bogotá). 152p.

Recibido. Mayo 26 de 2013

Aceptado: Octubre 2 de 2013

Como citar:

Aroca Araújo, A. 2013. Análisis de los diseños en los hipogeos del parque arqueológico de tierradentro, Cauca, Colombia. Rev. U.D.CA Act. \& Div. Cient. 16(2): 525-534. 